BULLETIN OF THE

AMERICAN MATHEMATICAL SOCIETY

Volume 81, Number 1, January 1975

\title{
UNIFORMLY TRIVIAL MAPS INTO SPHERES
}

\author{
BY ALLAN CALDER
}

Communicated by S. Eilenberg, September 19, 1974

A map (continuous function) is uniformly trivial if it is uniformly homotopic to a constant map. The universal covering $e: R \rightarrow S^{1}$ of the circle by the real line is an example of a map which is homotopically trivial but not uniformly so. For any space $X$ a map $f: X \rightarrow S^{1}$ is uniformly trivial if and only if there is a bounded map $g: X \rightarrow R$ such that $e g=f[E]$.

For spheres of higher dimension it has been shown that every map from euclidean $n$-space or $(n+1)$-space to the $n$-sphere, $S^{n}$, is uniformly trivial [C1], [C2]. Here we announce the following extensions of these results.

THEOREM 1. For $(X, A)$ a finite dimensional triangulable pair of spaces and $n>1$, a map $f:(X, A) \rightarrow\left(S^{n}, *\right), * \in S^{n}$, is uniformly trivial if and only if it is homotopically trivial.

THEOREM 2. For $X$ a contractible finite dimensional triangulable space and $Y$ a compact space, the fundamental group, $\pi_{1}(Y)$, of $Y$ being finite implies that every map from $X$ to $Y$ is uniformly trivial, but there exists uniformly nontrivial maps from $X$ to $Y$ if $X$ is noncompact and $\pi_{1}(Y)$ contains an element of infinite order.

If $(X, A)$ and $(Y, B)$ are pairs of completely regular hausdorff spaces, the two maps $f, g:(X, A) \rightarrow(Y, B)$ are uniformly homotopic if their extensions $\beta f, \beta g:(\beta X, \beta A) \rightarrow(\beta Y, \beta B)$ are homotopic. Here $\beta$ denotes the Stone-Čech compactification. For equivalent definitions see [D] and [ES].

Outline of PROOF of TheOREM 1. For $n \geqslant 1$ the free topological group $F$ on $S^{n}$ can be considered as a $\mathrm{CW}$-complex of finite type (i.e. the $m$-skeleton $F^{m}$ of $F$ is compact for each $m$ ), and that there is an embedding, $i: S^{n} \rightarrow F$, of $S^{n}$ as a subcomplex of $F$, which represents a generator of $\pi_{n}(F)$. This is "folklore" but can easily be deduced from the proof of Theorem 1 in $[\mathbf{H}]$.

Let $\left(E, p, S^{n+1}\right)$ be the fiber bundle over $S^{n+1}$ with fiber

AMS (MOS) subject classifications (1970). Primary 55D99; Secondary 54E60, $54 \mathrm{D35}$. 
$F$ and characteristic map $i$ [S, p. 97]. Since $i$ represents a generator of $\pi_{n}(F)$, the transgression $\Delta: \pi_{n+1}\left(S^{n+1}\right) \rightarrow \pi_{n}(F)$, in the homotopy sequence of the bundle, is an isomorphism. But $\Delta$ characterizes the bundle [S, p. 99] so the bundle is equivalent to the one obtained by Milnor's construction [M] with fiber $F$ and contractible total space. Hence $E$ is contractible.

Triangulate $S^{n+1}$ in such a way that the two hemispheres and an equatorial band are subcomplexes. Then $E$ can be given the structure of a CW-complex of finite type such that $p$ is a cellular map. Let $X$ be a simplicial complex and $f: X \rightarrow S^{n+1}$ a simplicial map. It can then be shown that if $f$ has a lift to $E$, i.e. $f$ is homotopically trivial, then there is a lift, $\varphi: X \rightarrow E$, of $F$ such that the image of the $m$-skeleton of $X$ is contained in the $(m+n)$-skeleton of $E$, i.e., $\varphi\left(X^{m}\right) \subset E^{m+n}$. But $E$ is of finite type so if $X$ is finite dimensional, $\varphi(X)$ is contained in a compact subset of $E$. This means that $\varphi$ can be extended to $\beta X$ to become a lift of $\beta f: \beta X$ $\rightarrow S^{n+1}$, and so $\beta f$ is homotopically trivial. In other words, $f$ is uniformly trivial.

The "if" part of the theorem now follows, since any map from a triangulable space to a finite simplicial complex is uniformly homotopic to a simplicial map, for a suitable triangulation of the domain. The "only if" part is trivial.

As an immediate corollary to Theorem 1 we get

Corollary. A finite dimensional triangulable pair, $(X, A)$, is $n$-coconnected, for $n>0$, if and only if its Stone-Čch compactification $(\beta X, \beta A)$ is n-coconnected.

Thus if $X$ is $n$-coconnected, $n>0$, and $Y$ is a compact $n$-connected space, every map from $X$ to $Y$ is uniformly trivial.

OUtLiNE OF PROOF OF THEOREM 2. If $\pi_{1}(Y)$ is finite, then the universal covering space of $Y$ is also compact and the result follows from the Corollary. If $X$ is noncompact it is not pseudocompact (pseudocompact + paracompact $=$ compact), and so there is a homotopically nontrivial map $u$ : $\beta X \rightarrow S^{1} \quad$ [D]. Let $v: S^{1} \rightarrow Y$ represent an element of infinite order in $\pi_{1}(Y)$. It can be shown that in singular homology, $u$ induces a nontrivial morphism and $v$ induces a monomorphism. Hence $v u$ is homotopically nontrivial.

It appears to be an open question as to whether every finitely presented infinite group contains an element of infinite order. If that conjecture is 
true, then for $X$ a noncompact finite dimensional triangulable space and $Y$ a compact space with the homotopy type of a $\mathrm{CW}$-complex, we would have that every map from $X$ to $Y$ is uniformly trivial if and only if $\pi_{1}(Y)$ is finite.

Details of the proofs will appear in [C3]. The author is grateful to E. H. Brown for several very useful conversations with regard to this work and to J. P. L. Hardy for most helpful information about free topological groups.

\section{REFERENCES}

C1. A. Calder, For $n>1$ any map $R^{n} \rightarrow S^{n}$ is uniformly homotopic to a constant, Nederl. Akad. Wetensch. Proc. Ser. A $75=$ Indag. Math. 34 (1972), 32-36. MR 46 \#4537.

C2. - On the cohomology of $\beta R^{n}$, Quart. J. Math. Oxford Ser. (3) 25 (1974), 385-394.

C3. Cohomology of finite covers (to appear).

D. C. H. Dowker, Mapping theorems for non-compact spaces, Amer. J. Math. 69 (1947), 200-242. MR 8, 594.

E. S. Eilenberg, Transformations continues en circonférence et la topologie du plan, Fund. Math. 26 (1936), 61-112.

ES. S. Eilenberg and N. Steenrod, Foundations of algebraic topology, Princeton Univ. Press, Princeton, N. J., 1952. MR 14, 398.

H. J. P. L. Hardy, The free topological group on a CW-complex (preprint).

M. J. W. Milnor, On the construction of FK, Lecture notes, Princeton University, 1956.

S. N. Steenrod, The topology of fiber bundles, Princeton Math. Ser., vol. 14, Princeton Univ. Press, Princeton, N. J., 1951. MR 12 \#522.

DEPARTMENT OF MATHEMATICS, BIRKBECK COLLEGE, UNIVERSITY OF LONDON, LONDON, ENGLAND 